\title{
Aspectos socio-económicos influyentes en el sector agrícola y su relación con la creación de empresas populares y solidarias de Quevedo
}

\section{Influential socio-economic aspects in the agricultural sector and its relation with the creation of popular and solidarity companies of Quevedo}

\footnotetext{
*Julia Fajardo Arriaga ${ }^{1}$, Lugarda Recalde Aguilar ${ }^{1}$, Mireya Flores Jaen ${ }^{1}$, Daniel Parra Gavilanes ${ }^{1}$, Acurio Rodríguez Carlos Javier ${ }^{l}$

${ }^{1}$ Universidad Técnica Estatal de Quevedo. Facultad de Ciencias Empresariales, Escuela de Gestión Empresarial. Campus Ing. Manuel Haz Alvares, km 1.5 vía Santo Domingo de Los Tsáchilas.

EC.120301.Quevedo,Ecuador.*jfajardo@uteq.edu.ec; 1recalde@uteq.edu.ec; mflores@uteq.edu.ec; dparra@uteq.edu.ec, cacuario@uteq.edu.ec.
}

Rec.: 12.02.2016. Acept.: 20.05.2016.

Publicado el 30 de diciembre de 2016

Rev. Cienc. Soc. y Econ. UTEQ (2016), 1(1): 107-116

\begin{abstract}
Resumen
El presente trabajo agrupa aspectos socio-económicos derivados de la agricultura en el cantón Quevedo, lo cual tiene estrecha relación con la creación de empresas populares y solidarias; cuyos resultados se ven reflejados en encuestas realizadas a agricultores de la referida zona, en la que se pudo precisar baja tasa de interés en la comercialización, insumos agropecuarios y préstamos. El objetivo de la investigación radica en evaluar los aspectos socio-económicos de la agricultura que están incidiendo de manera negativa en el sector agrícola a partir de la creación de empresas populares y solidarias en Quevedo. Los resultados obtenidos permitieron demostrar la necesidad del estudio para lograr los beneficios alcanzados respecto a la creación de las asociaciones, su estrategia productiva alcanzada y su capacidad de proyección hacia el mercado para el intercambio comercial su financiamiento, y la necesidad de generar ingresos para mejorar la calidad de vida del sector agrícola. Se realiza una investigación descriptiva - documental, a partir de la revisión de los referentes teóricos de investigadores, se aplica el método inductivodeductivo con la finalidad de solucionar la problemática planteada.
\end{abstract}


Palabras clave: intercambio comercial, financiamiento, producciones alcanzadas, calidad de vida e insumos agropecuarios

\begin{abstract}
The present work groups socio-economic aspects derived from the agriculture in the Quevedo canton, which has close relation with the creation of popular and solidarity companies; The results of which are reflected in surveys carried out on farmers in the said area, where a low rate of interest in the commercialization, agricultural inputs and loans could be determined. The objective of the research is to evaluate the socio-economic aspects of agriculture that are having a negative impact on the agricultural sector since the creation of popular and solidarity companies in Quevedo. The obtained results allowed to demonstrate the necessity of the study to achieve the benefits achieved in relation to the creation of the associations, its productive strategy reached and its capacity of projection to the market for the commercial exchange of its financing, and the need to generate income to improve the Quality of life of the agricultural sector. A descriptive documentary research is carried out, based on the review of the theoretical references of researchers, the inductive - deductive method is applied in order to solve the problem.
\end{abstract}

Keywords: trade, financing, production achieved, quality of life and agricultural inputs

\title{
Introducción
}

El Ecuador es un país privilegiado, debido a que cuenta con una situación geográfica y un suelo próspero para casi toda clase de cultivos lo que incide de forma favorable en una buena producción agrícola que ha convertido la agricultura una actividad generadora de fuente de ingreso (Barsky, 1984).

Desde lo argumentado, en el país se hace referencia a las redes de emprendimientos encaminadas a fomentar la cultura agrícola como una de las vías para alcanzar resultados favorables en el desarrollo de proyectos agropecuarios que llevan aparejados la sostenibilidad alimentaria (Salinas, 2012). 
La Economía Popular y Solidaria (EPS) constituye una alternativa consolidada para las capas menos favorecidas dentro de la economía ecuatoriana. La misma se desarrolla mediante la creación e implementación de emprendimientos, que en la generalidad de los casos no tienen una larga vida. A pesar de lo anterior el gobierno ecuatoriano fomenta facilidades para el desarrollo de dichos emprendimientos locales, (Boza, 2016).

La ciudad de Quevedo es considerada la nueva capital bananera de Ecuador, por lo cual tiene como una de sus actividades económicas la agricultura debido a que la población ocupada de Quevedo en el área rural es de 7854 habitantes que viene siendo un 96\% (INEC, 2010) del total de la población económicamente activa ubicada en la zona rural de la ciudad dedicada a la agricultura, cuya población aporta a los ingresos de la ciudad por lo cual la ciudad es conocida como el mayor centro económico y comercial de la provincia de Los Ríos, entregando sus productos agrícolas como: banano, café, cacao, palo de balsa, caucho, palma africana, frutales, soya, maíz, entre otros. Siendo esta una actividad de gran importancia estratégica como base fundamental para el desarrollo autosuficiente y riqueza de esta ciudad (Plan de desarrollo y ordenamiento territorial Los Ríos, sistema de planificación, 2012).

A pesar de que la ciudad, es muy activa en la agricultura hay ciertos aspectos que actualmente están afectando el crecimiento de esta actividad económica entre ellas el uso excesivo de plaguicidas que ocasionan daños a la salud humana, medio ambiente y al suelo, donde se cultivan los productos lo que conlleva a que cada vez se utilice menos cantidad de tierra para la producción, por ello es importante investigar cómo se encuentra la economía del sector agrícola, los factores negativos que estén incidiendo en la agricultura y su efecto a futuro para de ésta manera procurar mitigarlo o eliminarlo, y de ser positivo desarrollar las estrategias necesarias para tener una agricultura sustentable y sostenible, ( Bretón, 2003).

Esta investigación permite identificar los aspectos socio-económicos de la agricultura que están incidiendo en los resultados del sector agrícola con la creación de empresas populares y solidarias en Quevedo, para lo cual se requiere de un plan estratégico que resuelva a corto plazo la problemática planteada. 


\section{Materiales y Métodos}

Se realiza una investigación descriptiva - documental, a partir de la revisión de importantes aportes de investigadores clásicos, se emplea el método de induccióndeducción con la finalidad de identificar los indicadores que traen aparejados los resultados alcanzados en la investigación.

Se realizó la observación directa para conocer temas relacionados con la agricultura, la aportación económica que tiene este sector en la economía del país y la relación que tiene con las empresas populares y solidarias en el cantón Quevedo, así como para identificar los aspectos socioeconómicos de la agricultura, y su incidencia en las asociaciones agrícolas de Quevedo. Se obtuvo información valiosa del Ministerio de Agricultura Ganadería Acuacultura y Pesca MAGAP, del Centro de Agrícola de Quevedo, Asociaciones agrícolas.

Para efecto del cálculo de la muestra se consideraron 7854 personas dedicadas a la agricultura en la zona rural, y que tienen como actividad la agricultura. La ecuación aplicada fue de Santoyo-Cortés et al. (2000)

$$
\mathrm{n}=\frac{\mathrm{N} * \mathrm{z}_{\alpha}^{2} * \mathrm{p} * \mathrm{q}}{\mathrm{d}^{2} *(\mathrm{~N}-1) * \mathrm{z}_{\alpha}^{2} * \mathrm{p} * \mathrm{q}}
$$

$\mathrm{N}=$ Tamaño de la muestra

$\mathrm{Z}=$ Nivel de confianza

$\mathrm{P}=$ Éxito

$\mathrm{Q}=$ Fracaso

$\mathrm{D}=$ Margen de error

Siendo:

$\mathrm{N}=7854$

$\mathrm{z}_{\alpha}(90 \%)=1,64$

$\mathrm{p}=0.95$

$q=0.05$

$d=0,1$

$\mathrm{N}-1=7853$

Aplicación: 


$$
\mathrm{n}=\frac{7854 * 1,64_{\alpha}^{2} * 0,95 * 0,05}{0,1^{2} *(7854-1)+1.64_{\alpha}^{2} * 0.95 * 0.05}=\frac{1003}{2560}=392
$$

La muestra para realizar las encuestas es de 392 personas, las mismas fueron encuestadas para la realización de la investigación.

\section{Resultados}

El cuadro 1, demuestra que de los 392 encuestados el 51,02\% manifiestan que el problema socioeconómico más influyente que tiene la agricultura en Quevedo es la comercialización, el $25,51 \%$ opinaron que los insumos agrícolas y el 17,86 \% indicaron que los préstamos con bajas tasas de interés son sustancial para la producción.

\section{Cuadro 1. Aspectos socioeconómicos de la agricultura}

\begin{tabular}{lcc}
\hline \multicolumn{1}{c}{ Opción de respuesta } & Frecuencia absoluta & Porcentaje \\
\hline Comercialización & 200 & 51,02 \\
Sistema de riego & 10 & 2,55 \\
Préstamos con baja tasa de interés & 70 & 17,86 \\
Insumos agropecuarios & 100 & 25,51 \\
Todos & 12 & 3,06 \\
Total & 392 & $100 \%$ \\
\hline
\end{tabular}

Fuente: Encuesta a agricultores del Cantón Quevedo

El Cuadro 2, presenta las empresas Populares y Solidarias que están formadas y legalizadas de acuerdo a la ley, las mismas que están registradas en el Ministerio de Agricultura y Ganadería (MAGAP), en el Instituto de Economía Popular y Solidaria (IEPS) y en Centro Agrícola de Quevedo, las cuales se encuentran activas ya que todos los agricultores afiliados se preocupan por producir y por el bienestar de todos sus socios buscando estrategias mediante reuniones donde buscan la opinión de los agricultores que contribuyan a optimizar sus rentabilidad, en cuanto a la ubicación de las asociaciones 7 de ellas están en el cantón Quevedo que corresponde al 64\% mientras que 4 de las asociaciones están ubicadas en San Carlos con un 36\%. 
Cuadro 2. Empresas Populares y Solidarias del sector agrícola de Quevedo

\begin{tabular}{|c|c|c|c|}
\hline $\begin{array}{c}\text { Representante } \\
\text { legal }\end{array}$ & Cantón & Parroquia & Razón Social \\
\hline Troya Obdulia & Quevedo & San Carlos & $\begin{array}{l}\text { Asociación De Productores Agrícolas } \\
\text { Unidos Por El Agro }\end{array}$ \\
\hline Macías José & Quevedo & Quevedo & $\begin{array}{l}\text { Asociación De Productores Bananeros Del } \\
\text { Ecuador APROBANEC }\end{array}$ \\
\hline Veliz Andrés & Quevedo & Quevedo & $\begin{array}{l}\text { Asociación De Productores Agrícolas } \\
\text { Nuevos Horizontes }\end{array}$ \\
\hline Martínez Justo & Quevedo & San Carlos & $\begin{array}{l}\text { Asociación De Productores Agricolas } \\
\text { Pavon Chipe }\end{array}$ \\
\hline Arias Tito & Quevedo & Quevedo & $\begin{array}{l}\text { Corporación De Agricultores Avanzada } \\
\text { Alfarista }\end{array}$ \\
\hline Carriel Climaco & Quevedo & Quevedo & Centro Agrícola Cantonal De Quevedo \\
\hline $\begin{array}{l}\text { Carranza } \\
\text { Florentino }\end{array}$ & Quevedo & Quevedo & $\begin{array}{l}\text { Unión De Organizaciones Campesinas De } \\
\text { Quevedo }\end{array}$ \\
\hline $\begin{array}{l}\text { Ullon Juan } \\
\text { Carlos }\end{array}$ & Quevedo & San Carlos & $\begin{array}{l}\text { Asociación De Productores Agrícolas } \\
\text { Renace La Esperanza }\end{array}$ \\
\hline Moran Manuel & Quevedo & San Carlos & $\begin{array}{l}\text { Asociación De Productores Agrícolas } \\
\text { Fuerza Unidas }\end{array}$ \\
\hline Guerra Víctor & Quevedo & Quevedo & $\begin{array}{l}\text { Asociación De Agricultores Colonia Rafael } \\
\text { Correa Delgado }\end{array}$ \\
\hline Cedeño Jorge & Quevedo & Quevedo & $\begin{array}{l}\text { Asociación De Campesinos Narcisa De } \\
\text { Jesús }\end{array}$ \\
\hline
\end{tabular}

Fuente: Ministerio de Agricultura Ganadería Acuacultura y Pesca (MAGAP)

Mediante el cuadro 3. Se aprecia que el mayor número de socios registrados se encuentra en la Asociación de Agricultores Colonia Rafael Correa Delgado con un número de 99 personas, mientras que Asociación de productores agrícolas Unidos por el Agro, cuenta con 10 afiliados, de las cuales 6 de ellas están registradas en el MAGAP, 3 en el IEPS y 2 en el Centro Agrícola, además se puede notar que las reuniones realizadas por la empresa están en promedio de 1 a 2 por mes. 


\section{Cuadro 3. Existencia y funcionamiento legal de las asociaciones encuestadas}

\begin{tabular}{|c|c|c|c|}
\hline Asociaciones & No de socios & Registros & $\begin{array}{c}\text { Reuniones } \\
\text { /mes }\end{array}$ \\
\hline $\begin{array}{l}\text { Asociación de productores Agrícolas } \\
\text { Unidos por El AGRO }\end{array}$ & 10 & MAGAP & 2 \\
\hline $\begin{array}{l}\text { Asociación de productores bananeros del } \\
\text { Ecuador APROBANEC }\end{array}$ & 15 & MAGAP & 2 \\
\hline $\begin{array}{l}\text { Asociación de Productores Agricolas } \\
\text { Nuevos Horizontes }\end{array}$ & 40 & MAGAP & 2 \\
\hline $\begin{array}{l}\text { Asociación de Productores Agrícolas } \\
\text { Pavón Chipe }\end{array}$ & 39 & MAGAP & 2 \\
\hline $\begin{array}{l}\text { Corporación de Agricultores Avanzada } \\
\text { Alfarista }\end{array}$ & 44 & MAPGAP & 2 \\
\hline Centro Agrícola Cantonal de Quevedo & 62 & $\begin{array}{l}\text { CENTRO } \\
\text { AGRICOLA }\end{array}$ & 3 \\
\hline $\begin{array}{l}\text { Unión de Organizaciones Campesinas de } \\
\text { Quevedo }\end{array}$ & 22 & MAGAP & 1 \\
\hline $\begin{array}{l}\text { Asociación de Productores Agrícolas } \\
\text { Renace La Esperanza }\end{array}$ & 29 & IEPS & 1 \\
\hline $\begin{array}{l}\text { Asociación de Productores Agrícolas } \\
\text { Fuerza Unidas }\end{array}$ & 11 & IEPS & 1 \\
\hline $\begin{array}{l}\text { Asociación de Agricultores Colonia } \\
\text { Rafael Correa Delgado }\end{array}$ & 99 & IEPS & 1 \\
\hline $\begin{array}{l}\text { Asociación de Campesinos Narcisa de } \\
\text { Jesús }\end{array}$ & 27 & $\begin{array}{l}\text { CENTRO } \\
\text { AGRICOLA }\end{array}$ & 1 \\
\hline
\end{tabular}

Fuente: Ministerio de Agricultura Ganadería Acuacultura y Pesca (MAGAP)

\section{Discusión}

(Quimi, 2013), ofrece una visión clara de la realidad de los agricultores, al respecto señala que Ecuador es uno de los países que tiene un clima excelente para la producción de las tierras, sin embargo se encuentran falencias en las condiciones de vida de los agricultores y en la poca importancia por parte de los políticos de turno, debido a que en el campo se 
necesita sistemas de riego, electricidad, transporte, lastrado, medicamentos, doctores, tecnología. Etc. Esto contribuye a que los agricultores opten por dejar de producir y busquen oportunidades en el sector urbano.

Por otra parte, el estudio realizado en el Proyecto de Investigación según los resultados en la encuesta aplicada concuerda con (Quimi, 2013), ya que el 51,02\% de los agricultores opinan que se deben considerar estrategias para comercializar su producción, el $25.51 \%$ manifestaron que otro factor importante son los insumos agrícolas, además de esto cabe recalcar que el $76.53 \%$ de las personas dedicadas a la agriculturas y encuestadas supieron decir que en sus asociaciones les hace falta tecnología, entre ellas, maquinarias para la producción.

El estudio realizado por (MAGFOR, 2009), sostiene que el sector agrícola es considerado como prioridad dentro del Plan Nacional de Desarrollo Humano (PNDH) de países latinoamericanos, y de los cuales Ecuador no está exento, se precisa la implementación de programas que fomenten la asociación de los pequeños y medianos productores con el fin de que ellos tengan asistencia técnica, créditos y semillas de calidad para que los agricultores alcancen altos niveles de producción de calidad.

Si bien es cierto que en el desarrollo de la presente investigación se muestran los resultados de los agricultores que forman parte de la Institución, se ha podido considerar los beneficios que aportan al gobierno, es por ello que este estudio se identifica en gran parte con lo mencionado por MAGFOR 2009, sin embargo en las encuestas realizadas el $63.78 \%$ de los agricultores encuestados manifestaron que en la actualidad no han recibido apoyo por parte del gobierno, lo que ha provocado que entre ellos mismos hayan buscado estrategias para la comercialización de sus productos y darse el apoyo entre socios respectivamente.

\section{Conclusiones}

El sector agrícola, es un componente principal en la economía Quevedeña, reflejándose que entre los aspectos socio-económicos influyentes en la agricultura del cantón Quevedo, la comercialización, los insumos agropecuarios, y los préstamos con baja tasa de interés. 
La creación de las empresas populares y solidarias, han contribuido al mejoramiento de la producción, al intercambio y comercialización, así como a la búsqueda de fuentes de financiamiento para que los productos y bienes logren satisfacer las necesidades de la comunidad del cantón Quevedo.

Se ha precisado la necesidad de involucrar en este proceso a docentes y estudiantes universitarios para contribuir al mejoramiento de los problemas socioeconómicos en base al Plan Nacional del Buen Vivir establecidos legalmente en la Constitución de la República de Ecuador.

\section{Referencias bibliográficas}

Barsky, O., (1984). Acumulación campesina en el Ecuador, FLACSO-Ecuador, Quito

Boza, J. (2015). Atenciones teóricas de la economía popular y solidaria (EPS). Revista OIDLES, 9(18):19. ISSN 1988-2483.

Boza, J. (2015). Piloto de emprendimientos turísticos y su aporte en el desarrollo del ecoturismo sostenible. Revista Res Non Verba. (7), 65-76. ISSN 1390-6968.

Bretón, V., (2003). "Desarrollo rural y etnicidad en las Tierras Altas del Ecuador”, en V. Bretón y F. García, editores, Estado, etnicidad y movimientos sociales en América Latina. Ecuador en crisis, Icaria, Barcelona, p .217-256

CFN. (2014). http://www.finanzaspopulares.gob.ec/wpcontent/uploads/Eventos/Encuen tro\%20Nacional\%20de\%20Finanzas\%20Populares.

IEPP. (2014). Economia Popular y Solidaria y Ley Orgànica.

INEC. (2013). Encuesta de Superficie y Producción Agropecuaria Continua. Ed. INEC Ecuador.

INEC. (2010). Censo realizado en el año 2010.

Ministerio Agropecuario y Forestal. (MAGFOR). (2009). Plan Institucional. Nicaragua.

Secretaría de Educación Superior, Ciencia, Tecnol. (2014). http://www.educacionsuperior.gob.ec/el-sector-agricola-y-de-produccion-dealimentos-se-beneficiara-con-investigacion-y-tecnologia/. 
Salinas, F., Osorio, L. (2012). Emprendimiento y Economía Social, oportunidades y efectos en una sociedad en transformación. Revista CIRIEC. (75):129-151. ISSN 0213-8093.

Plan de desarrollo y ordenamiento territorial Los Ríos. (2012). Sistema de planificación.

Quimi, V. (09 de 01 de 2013). http://elproductor.com/2013/01/09/la-realidad-de-losagricultores-y-de-la-agricultura-ecuatoriana/.

RIMISP, (2012). Centro Latinoamericano para el desarrollo rural, Productividad del sector ecuatoriano.

Santoyo-Cortés, H., Ramírez-Moreno, P., Suvedi, M. 2000. Manual para la evaluación de programas de desarrollo rural. Centro de Investigaciones Económicas, Sociales y Tecnológicas de la Agroindustria y la Agricultura Mundial- Universidad Autónoma Chapingo-Instituto Nacional de Capacitación Rural. México, D.F. 250 p. 